\title{
A case of osmotic demyelination syndrome (ODS) associated with undiagnosed Cushing's disease
}

\begin{abstract}
Here we describe a case of undiagnosed Cushing disease, leading to diabetes mellitus, hypoglycemic coma, and central pontine myelinolysis. A 24 year-old woman presented with several weeks of progressive agitation and confusion. On physical exam there was buffalo hump, moon facies, abdominal striae, and centripetal obesity. MRI revealed demyelinating lesions in the central pontine region consistent with osmotic demyelination syndrome (ODS). Overnight $8 \mathrm{mg}$ dexamethasone suppression test was $2.5 \mathrm{mcg} / \mathrm{dL}$, which confirmed the diagnosis of Cushing disease. This case adds to the literature suggesting that ODS can occur directly from acute severe hypoglycemia and hypokalemia, due to dysregulation of the sodium-potassium-ATPase pump.
\end{abstract}

Volume 6 Issue 6 - 2018

\section{Cameron Page, Evariste Mushuru, Anthony Bazatsinda}

College of Medicine and Health Sciences, University of Rwanda, USA

\author{
Correspondence: Cameron Page, Assistant Professor of \\ Medicine, College of Medicine and Health Sciences, University \\ of Rwanda, SUNY Downstate Medical Center, 450 Clarkson \\ Avenue, Brooklyn, NY I I203, \\ Email cameron.page@downstate.edu
}

Received: October 26, 2017 | Published: December 20, 2018

\section{Introduction}

Osmotic Demyelination Syndrome (ODS, formerly known as Central Pontine Myelinolysis) is a well-described complication of overly rapid correction of hyponatremia, resulting in demyelination of the central pontine area of the brainstem. Clinical manifestations vary from tremors and confusion to quadriplegia. There are reports of CPM occurring secondary to acute hypoglycemia. ${ }^{1}$

Cushing syndrome is the clinical constellation of signs and symptoms that result from overproduction of cortisol in the adrenal gland or an ectopic source. On history patients often report amenorrhea, weakness, impotence, hirsutism, polydipsia and polyuria, headache, and abdominal pain. Physical exam findings include dorsicocervical fat pad, facial plethora, centripetal obesity, abdominal striae, hypertension, and glucose intolerance. Multiple reviews have noted that neuropsychological changes (depression, anxiety, panic disorder) are present in more than $50 \%$ of patients with Cushing syndrome, and can often be the presenting complaint. ${ }^{2,3}$

The excess cortisol that leads to Cushing syndrome can be produced in three ways: 1) tumors, especially lung cancer, produce ACTH, 2) a functional adenoma that produces ACTH can be located in the posterior pituitary ("classic" Cushing disease) or 3) the adenoma can be in an ectopic location.

Here we report a case of a patient with undiagnosed Cushing disease for many years that was complicated by secondary diabetes leading to an episode of severe hypoglycemia and central pontine myelinolysis.

\section{Case report}

A 24 year-old woman presented with confusion and agitation that had been progressive for several weeks. Five years earlier she was diagnosed with diabetes mellitus, and one year after that she experienced multiple episodes of severe hypoglycemia, one of which led to diabetic coma. After regaining consciousness her family stated that she grew agitated and confused, and she was admitted to a psychiatric referral hospital where she was placed on depakote and flupentixol. Since that time she had decreased consciousness and impaired ability to perform ADLs. She also complained of generalized body swelling and loss of menstruation since the start of her illness. Three years ago she was diagnosed with hypothyroidism and hypertension, and treated with anti-hypertensive medications and levothyroxine. She reported blurred vision and was diagnosed with bilateral cataracts. She also complained of numbness and tingling involving both upper limbs and lower limbs. She is a survivor of the 1994 genocide, and she has annual visual and auditory hallucinations in the weeks surrounding the commemoration of the genocide. Her medications at the time of admission were depakote, rapid and longacting insulin, nifedipine, captopril, and atenolol.

On physical exam, her blood pressure was $180 / 124 \mathrm{mmHg}$, pulse 95, Temp 36.7 Celsius, O2 95\% on RA. She was ill-looking and confused, but not in respiratory distress. She had generalized anasarca involving the face, abdomen and $2+$ pitting edema of the lower limbs to the knee. Strength was $5 / 5$ throughout and sensation was conserved. There was buffalo hump, moon facies, significant abdominal striae, and centripetal obesity.

MRI revealed demyelinating lesions in the central pontine region consistent with osmotic demyelination syndrome (thin black arrow, Figure 1). The 24-hour urine free cortisol was $415 \mathrm{mcg} / \mathrm{dL}$ (normal range $10-100 \mathrm{mcg} / \mathrm{d}$ ). Overnight $8 \mathrm{mg}$ dexamethasone suppression test was $2.5 \mathrm{mcg} / \mathrm{dL}$, which confirmed the diagnosis of Cushing disease. Her neuroleptic medications were held, and over the next two weeks she regained alertness and lucidity.

\section{Discussion}

Here we report a rare clinical presentation of a pituitary microadenoma, diagnosed by urine and serum cortisol levels but not seen on brain MRI. Although uncommon, there are cases of pituitary microadenomas not visible on MRI., ${ }^{4,5}$ We performed two steroid suppression tests, and the urine and serum cortisol results definitively made the diagnosis of Cushing disease.

$\mathrm{CT} / \mathrm{MRI}$ imaging is not required to make a diagnosis of Cushing disease. A study of 118 patients who underwent transphenoidal surgery for pituitary adenomectomy sought to determine the prognostic importance of brain MRI for Cushing disease. ${ }^{5}$ They included 75 patients with adenoma visible on MRI, and 43 patients without visible MRI findings. No statistical difference was found between MRI- 
positive and MRI-negative groups with regard to either post-operative remission (85.3\% vs $74.4 \%$ ) or frequency of adenoma confirmation on pathology exam $(94.7 \%$ vs $88.4 \%)$. Thus we conclude that cortisol level in the urine and/or blood is the most reliable non-invasive approach to definitely diagnose Cushing disease.

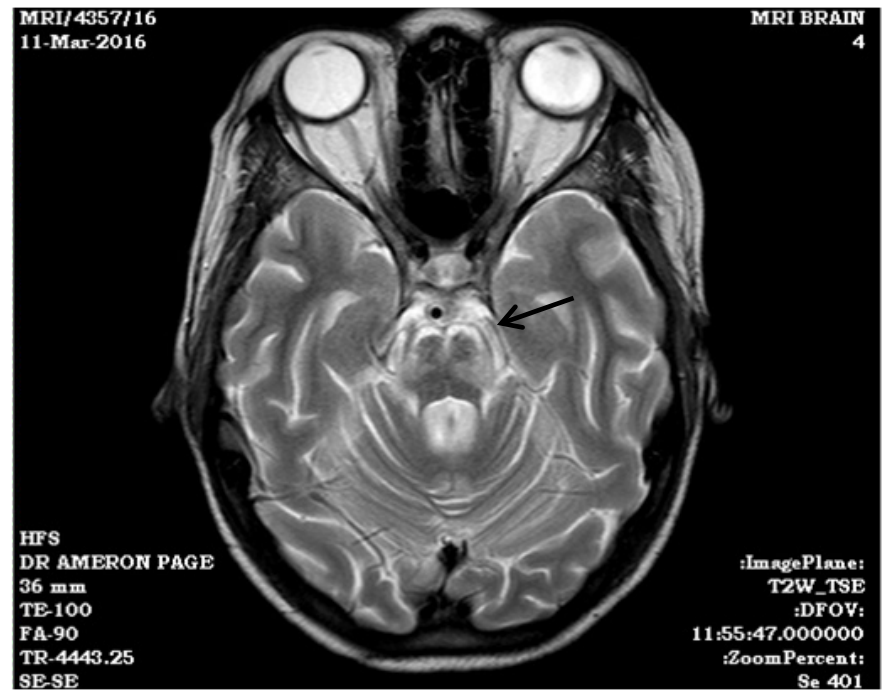

A

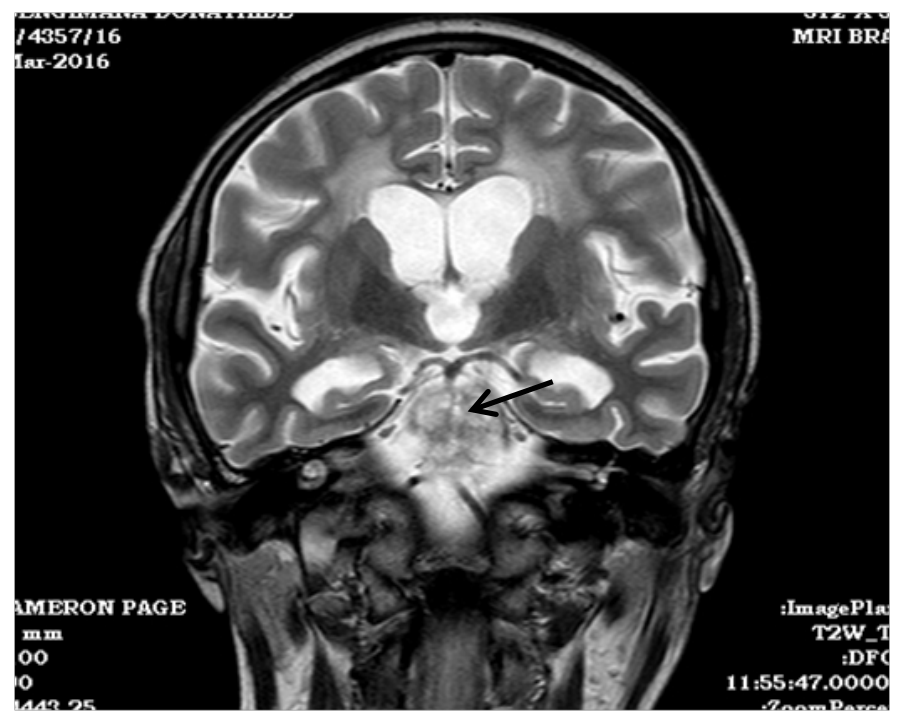

B

Figure IA \& B MRI revealed demyelinating lesions in the central pontine region.

The finding of Osmotic Demyelination Syndrome (ODS) is a unique feature of this case. Development of ODS occurred years prior to our contact with the patient, so we have limited ability to determine with confidence the antecedent injury that triggered ODS. Since the first description of ODS in 1959, (6) it has been clearly established that overly rapid correction of severe hyponatremia (below $120 \mathrm{mg}$ / $\mathrm{dL}$ ) is responsible. ${ }^{7-9}$

Some parts of the etiology of ODS are clear, while others remain controversial. It has been established that sodium plays an important role in osmotic homeostasis, and when $\mathrm{Na}+$ goes below 120 for more than 48 hours there is a compensatory influx of other osmolytes to protect neurons. These alternative osmolytes are not by themselves harmful: slow correction of hyponatremia allows them to efflux from the intracellular space without any damage done. The reason rapid correction of hyponatremia is harmful is that sodium crosses the cell membrane much faster than these alternative osmolytes. The overaccumulation of osmolytes inside the cells causes cell shrinkage and dehydration.

What is still not fully elucidated is the specific mechanism by which this cell shrinkage leads to myelinolysis and the subsequent neurological manifestations. Hypotheses include a separation of the glia from the neuron, secondary to the shrinking of the neuron, as well as direct apoptosis of the oligodendrocytes caused by the complement activation and the metabolic stress of dehydration..$^{10}$ There is also data suggesting a primary role of the dysregulation of the sodiumpotassium-ATPase pump in the cell membrane. ${ }^{11}$

If the sodium-potassium-ATPase hypothesis is correct, it would be consistent with cases of ODS occurring among patients with diabetes, such as this case. It is possible that the primary insult may have been dysregulation of potassium caused by inappropriate insulin injection. One review recommends correcting potassium imbalance prior to sodium imbalance, if the patient is neurologically stable. ${ }^{12}$

Certain features of the history do suggest hypokalemia could have played a role in the development of ODS in this patient. She had recently been diagnosed with diabetes mellitus and was taking insulin. The family reported that in the early months of her diagnosis they did not fully understand the nature of the illness, with respect to carbohydrate intake and the need to balance glycemia with her basalbolus insulin administration. Rather, the patient's family indicated that they learned to use insulin on a "trial-and-error" basis. It was during this learning phase that the patient developed the worst of her episodes of hypoglycemic encephalopathy.

Alternatively, excessive free water consumption may have been the primary trigger for ODS. This patient had glucose intolerance secondary to undiagnosed Cushing disease, and many patients with hyperglycemia and DKA develop polydipsia. We hypothesize that due to polydipsia in the setting of hyperglycemia, she overcompensated with massive free water consumption, which led to hyponatremia.

Furthermore, it is possible that interaction with the healthcare system exacerbated this patient's hyponatremia. She went to a rural Hospital in obtundation with undiagnosed Cushing disease. Due to her functional adenoma she was unable to regulate her sodium levels properly, and if the medical team gave her large amounts of IV fluids for suspected septic shock, they may have worsened her hyponatremia.

Due to this patient's presentation with features suggestive of mental illness (psychosis, agitation, confusion) she was incorrectly treated with psychoactive compounds for many years. In a low-resource setting such as Rwanda, where urine and serum cortisol levels are not routinely available and must be sent to specialized laboratories, it is understandable that the diagnosis would be delayed. The added burden of disease suffered by the patient and her family due to this delay, however, should be a reminder to us to continually revisit the diagnosis and be willing to reassess if the patient is not improving.

\section{Ethical Approval}

Informed consent was obtained from the patient and family members referenced in this case report. Approval from Institutional Review Board (IRB) is not applicable for this case report. 


\section{Contributorship}

EM wrote the first draft of the article. AB obtained supplementary information. EM and $\mathrm{AB}$ obtained patient and family consent. All authors reviewed and edited the manuscript and approved the final version of the manuscript. $\mathrm{CP}$ is the senior member of the team and supervisor of the other authors.

\section{Acknowledgements}

We would like to thank the patient and her family for her cooperation with this case report, as well as the University Teaching Hospital of Kigali (UTHK) for assistance in collecting supplementary materials. Special thanks to the Radiology Department for assistance with MRI interpretation and image capture.

\section{Declaration of competing interests}

None.

\section{Funding}

None.

\section{References}

1. Madey JJ, Hannah JA, Lazaridis C. Central pontine myelinolysis following acute hypoglycemia. Clin Neurol Neurosurg. 2013;115(10):2299-2300.

2. Kelly W. Psychiatric Aspects of Cushing Syndrome. QJM. 1996;89(7):543.

3. Haskett F. Diagnostic Categorization of Psychiatric Disturbance in Cushing 's syndrome. Am J Psychiatry. 1985;142(8):911-916.
4. Lim JH, Kim SJ, Jung MK, et al. A patient with Cushing disease lateralizing a pituitary adenoma by inferior petrosal sinus sampling using desmopressin: a case report. Ann Pediatr Endocrinol Metab. 2016;21(1):43-46.

5. Feng M, Bao X, Lu L, et al. Diagnosis and surgical management in patients with Cushing disease with negative magnetic resonance imaging. Europe PMC. 2015;95(39):3213-3216.

6. Adams RD, Victor M ME. Central pontine myelinolysis: a hitherto undescribed disease occurring in alcoholic and malnourished patients. AMA Arch Neurol Psychiatry. 1959;2:81(2)-82.

7. Mascarenhas J V, Jude EB. Central pontine myelinolysis: electrolytes and beyond. BMJ Case Rep. 2014; doi: 10.1136/bcr-2013-203516.

8. Singh TD, Fugate JE, Rabinstein AA. Central pontine and extrapontine myelinolysis: a systematic review. Eur J Neurol. 2014;21(12):14431450 .

9. Kumar S, Fowler M, Toledo E JS. Central pontine myelinolysis, an update. Neurol Res. 2006;28(3):360-366.

10. Baker EA, Tian Y, Adler S, et al. Blood-brain barrier disruption and complement activation in the brain following rapid correction of chronic hyponatremia. Exp Neurol. 2000;165(2):221-230.

11. Ashrafian H, Davey P. A review of the causes of central pontine myelinosis: Yet another apoptotic illness? Eur J Neurol. 2001;8(2):103109.

12. Lohr JW. Osmotic demyelination syndrome following correction of hyponatremia: Association with hypokalemia. Am J Med. 1994;96(5):408-413. 